\title{
Finite Dimension in Associative Rings
}

\author{
SATYANARAYANA BHAVANARI \\ Department of Mathematics, Acharya Nagarjuna University, Nagarjuna Nagar - \\ 522 510, A.P, India \\ e-mail : bhavanari2002@yahoo.co.in \\ NAGARAJu DASARI \\ Department of Mathematics, Acharya Nagarjuna University, Nagarjuna Nagar - \\ 522 510, A. P, India \\ e-mail : dasari.nagaraju@gmail.com \\ Balamurugan Kuppareddy Subramanyam \\ Department of Mathematics, R. V. R and J. C. College of Engineering, Chow- \\ davaram, Guntur - 522 019, A. P, India \\ e-mail : muruganbalaks@yahoo.co.in \\ Godloza Lungisile \\ Department of Mathematics, Walter Sisulu University, Umtata, South Africa \\ e-mail : lgodloza@wsu.ac.za
}

Abstract. The aim of the present paper is to introduce the concept "Finite dimension" in the theory of associative rings $\mathrm{R}$ with respect to two sided ideals. We obtain that if $\mathrm{R}$ has finite dimension on two sided ideals, then there exist uniform ideals $U_{1}, U_{2}, \cdots, U_{n}$ of $\mathrm{R}$ whose sum is direct and essential in $\mathrm{R}$. The number $\mathrm{n}$ is independent of the choice of the uniform ideals $U_{i}$ and 'n' is called the dimension of $\mathrm{R}$.

\section{Introduction}

The dimension of a vector space is defined as the number of elements in the basis. One can define a basis of a vector space as a maximal set of linearly independent vectors or a minimal set of vectors which span the space. The former when generalized to modules over rings become the concept of Goldie dimension. Goldie proved a structure theorem for modules which states that "a module with finite Goldie dimension (FGD, in short) contains a finite number of uniform submodules $U_{1}, U_{2}, \cdots, U_{n}$ whose sum is direct and essential in M". The number $\mathrm{n}$ obtained here is independent of the choice of $U_{1}, U_{2}, \cdots, U_{n}$ and it is called as Goldie dimension of M. The concept Goldie dimension in Modules was studied by several authors

Received July 6, 2006.

2000 Mathematics Subject Classification: 16B99, 16D25, 16D70, 16P99.

Key words and phrases: ring, two sided ideal, essential ideal, uniform ideal. 
like Reddy, Satyanarayana, Syam Prasad, Nagaraju (refer [4], [5], [6]).

If we consider ring as a module over itself, then the existing literature tells about dimension theory for ideals (i.e., two sided ideals) in case of commutative rings; and left (or right) ideals in case of associative (but not commutative) rings. So at present we can understand the structure theorem for associative rings in terms of one sided ideals only (that is, if R has FGD with respect to left (right) ideals, then there exist $\mathrm{n}$ uniform left (or right) ideals of $\mathrm{R}$ whose sum is direct and essential in $\mathrm{R}$ ). This result cannot say about the structure theorem for associative rings in terms of two sided ideals. So to fill the gap, we prove the structure theorem for associative rings with respect to two sided ideals.

Throughout the paper $\mathrm{R}$ denotes an associative ring (need not be commutative). The paper is divided into three sections. In Secton-2 we introduce and study the concepts: complement, essential with respect to two sided ideals of R. In Section-3, we introduce the concept: uniform ideal and study few fundamental results which are useful in Section-4. In Section-4, we introduce the concept "finite dimension". We obtain an equivalent condition for an associative ring $\mathrm{R}$ to have finite dimension, which is used in the later part. Finally, we prove the main theorem: If an ideal $\mathrm{H}$ has FDIR, then there exist uniform ideals $U_{1}, U_{2}, \cdots, U_{n}$ of $\mathrm{R}$ whose sum is direct and essential in $\mathrm{H}$. The number is independent of the choice of the uniform ideals $U_{i}, 1 \leq \mathrm{i} \leq \mathrm{n}$. This number $\mathrm{n}$ is called the dimension of $\mathrm{H}$ and we denote it by $\operatorname{dim}$ $\mathrm{H}$.

Let $\mathrm{R}$ be a fixed (not necessarily commutative) ring. We write $\mathrm{K} \unlhd \mathrm{R}$ to denote ' $\mathrm{K}$ is an ideal of $\mathrm{R}$ '. We use the term "ideal" for "two sided ideal". The ideal generated by an element $\mathrm{a} \in \mathrm{R}$ is denoted by $\langle a\rangle$. We do not include the proofs of some results when they are easy or straight forward verification.

\section{Essential ideals}

We start this section with the following definition.

Definition 2.1. Let I, J be two ideals of $\mathrm{R}$ such that $\mathrm{I} \subseteq \mathrm{J}$.

(i) We say that I is essential (or ideal essential) in $\mathrm{J}$ if it satisfies the following condition: $\mathrm{K} \unlhd \mathrm{R}, \mathrm{K} \subseteq \mathrm{J}, \mathrm{I} \cap \mathrm{K}=(0)$ imply $\mathrm{K}=(0)$.

(ii) If $\mathrm{I}$ is essential in $\mathrm{J}$ and $\mathrm{I} \neq \mathrm{J}$, then we say that $\mathrm{J}$ is a proper essential extension of I. If I is essential in J, then we denote this fact by $\mathrm{I} \leq_{e} \mathrm{~J}$.

Definition 2.2. If $K \unlhd R, A \unlhd R$ and $K$ is a maximal element in $\{\mathrm{I} / \mathrm{I} \unlhd \mathrm{R}, \mathrm{I} \cap$ $\mathrm{A}=(0)\}$, then we say that $\mathrm{K}$ is a complement of $\mathrm{A}$ (or a complement in $\mathrm{R}$ ).

Note 2.3. Let $\mathrm{I}$ and $\mathrm{J}$ be ideals of $\mathrm{R}$.

(i) $\mathrm{I} \leq_{e} \mathrm{~J} \Leftrightarrow \mathrm{I} \cap \mathrm{K}=(0), \mathrm{K} \unlhd \mathrm{R} \Rightarrow \mathrm{J} \cap \mathrm{K}=(0)$.

(ii) $\mathrm{B}$ is a complement in $\mathrm{R} \Leftrightarrow$ there exists an ideal $\mathrm{A}$ of $\mathrm{R}$ such that $\mathrm{B} \cap \mathrm{A}=(0)$ and $K^{1} \cap A \neq(0)$ for any ideal $K^{1}$ of $\mathrm{R}$ with $\mathrm{B} \varsubsetneqq K^{1}$. In this case $\mathrm{B}+\mathrm{A} \leq_{e} \mathrm{R}$.

[Verification: Suppose $\mathrm{B}$ is a complement in $\mathrm{R} \Rightarrow \mathrm{B}$ is complement of an ideal A of $R \Rightarrow B$ is a maximal ideal of $R$ with respect to the property $B \cap A=(0)$. Suppose 
that $K^{1} \unlhd \mathrm{R}$ with $\mathrm{B} \subsetneq K^{1}$. If $K^{1} \cap \mathrm{A}=(0)$, then since $\mathrm{B}$ is maximal with respect to $\mathrm{B} \cap \mathrm{A}=(0)$ we get $K^{1}=\mathrm{B}$, a contradiction. Therefore $K^{1} \cap A \neq(0)$. The converse is clear.

Now we show that $\mathrm{B}+\mathrm{A} \leq_{e} \mathrm{R}$. Let $\mathrm{J} \unlhd \mathrm{R}$ such that $(\mathrm{B}+\mathrm{A}) \cap \mathrm{J}=(0)$. Let $\mathrm{x} \in(\mathrm{B}+\mathrm{J}) \cap \mathrm{A} \Rightarrow \mathrm{x}=\mathrm{b}+\mathrm{j} \in \mathrm{A}$ where $\mathrm{b} \in \mathrm{B}$ and $\mathrm{j} \in \mathrm{J} \Rightarrow \mathrm{b}-\mathrm{x}=-\mathrm{j} \in(\mathrm{B}$ $+\mathrm{A}) \cap \mathrm{J}=(0) \Rightarrow \mathrm{j}=0 \Rightarrow \mathrm{x}=\mathrm{b} \in \mathrm{A} \cap \mathrm{B}=(0)$. Therefore $(\mathrm{B}+\mathrm{J}) \cap \mathrm{A}=$ (0). Since $B$ is maximal with respect to the property that $B \cap A=(0)$, we have that $\mathrm{B}+\mathrm{J}=\mathrm{B}$. Now $\mathrm{J} \subseteq \mathrm{B} \subseteq \mathrm{B}+\mathrm{A} \Rightarrow \mathrm{J}=\mathrm{J} \cap(\mathrm{B}+\mathrm{A})=(0)$. Thus $\left.\mathrm{B}+\mathrm{A} \leq_{e} \mathrm{R}\right]$.

(iii) If $\mathrm{A} \cap \mathrm{B}=(0)$, and $\mathrm{C}$ is an ideal of $\mathrm{R}$ which is maximal with respect to the property $\mathrm{C} \supseteq \mathrm{A}$ and $\mathrm{C} \cap \mathrm{B}=(0)$, then $\mathrm{C} \oplus \mathrm{B}$ is essential in $\mathrm{R}$. Moreover, $\mathrm{C}$ is a complement of B containing A. [Verification: Follows from (ii)].

Result 2.4. (i) The intersection of finite number of essential ideals is essential;

(ii) If $I, J, K$ are ideals of $R$ such that $I \leq_{e} J$, and $J \leq_{e} K$, then $I \leq_{e} K$;

(iii) $I \leq_{e} J \Rightarrow I \cap K \leq_{e} J \cap K$;

(iv) If $I \subseteq J \subseteq K$, then $I \leq_{e} K$ if and only if $I \leq_{e} J$, and $J \leq_{e} K$; and

(v) If $R_{1}, R_{2}$ are two rings, $f: R_{1} \rightarrow R_{2}$ is a ring isomorphism, and $A$ is an ideal of $R_{1}$, then $A \leq_{e} R_{1} \Leftrightarrow f(A) \leq_{e} R_{2}$.

Proof. Proof for (i) to (iv) is a straight forward verification.

(v) Suppose $\mathrm{A} \leq_{e} R_{1}$. We have to show that $\mathrm{f}(\mathrm{A}) \leq_{e} R_{2}$. Let $\mathrm{B}$ be an ideal of $R_{2}$ such that $\mathrm{f}(\mathrm{A}) \cap \mathrm{B}=(0)$. Now $\mathrm{A} \cap f^{-1}(B)=(0)$. Since $\mathrm{A}$ is essential in $R_{1}$, we have $f^{-1}(B)=(0)$ and so $\mathrm{B}=f\left(f^{-1}(B)\right)=(0)$. Thus $\mathrm{f}(\mathrm{A}) \leq_{e} R_{2}$. If $\mathrm{f}(\mathrm{A}) \leq_{e} R_{2}$. The other part is similar.

Note 2.5 (Refer page 158 of [1]). If $\mathrm{R}$ is a ring, $\mathrm{a} \in \mathrm{R}$, then $\langle a\rangle=\{\mathrm{ra}+\mathrm{as}+$ $\left.\mathrm{na}+\sum_{i=1}^{k} r_{i} a s_{i} / \mathrm{k} \in \mathrm{Z}, \mathrm{n} \in \mathrm{Z}, \mathrm{k} \geq 0, \mathrm{r}, \mathrm{s}, s_{i}, r_{i} \in \mathrm{R}\right\}$.

Remark 2.6. If $\mathrm{a}, \mathrm{b} \in \mathrm{R}$ and $\mathrm{x} \in\langle a\rangle$, then there exists $\mathrm{y} \in\langle b\rangle$ such that $\mathrm{x}$ $+\mathrm{y} \in\langle a+b\rangle$.

[Verification: Since $\mathrm{x} \in\langle a\rangle$, by above Note 2.5, it follows that $\mathrm{x}=\mathrm{ra}+$ as $+\mathrm{na}+\sum_{i=1}^{k} r_{i} a s_{i}$. If $\mathrm{y}=\mathrm{rb}+\mathrm{bs}+\mathrm{nb}+\sum_{i=1}^{k} r_{i} b s_{i} \in\langle b\rangle$, then $\mathrm{x}+\mathrm{y}=\mathrm{r}(\mathrm{a}$ $+\mathrm{b})+(\mathrm{a}+\mathrm{b}) \mathrm{s}+\mathrm{n}(\mathrm{a}+\mathrm{b})+\sum_{i=1}^{k} r_{i}(a+b) s_{i} \in<a+b>$.]

Lemma 2.7. (i) $L_{1}, L_{2}, K_{1}, K_{2}$ are ideals of $R$ such that $L_{i} \subseteq K_{i}$ for $i=1$, 2 and $K_{1} \cap K_{2}=(0)$. Then $L_{1} \leq_{e} K_{1}$ and $L_{2} \leq_{e} K_{2} \Leftrightarrow L_{1}+L_{2} \leq_{e} K_{1}+K_{2}$; and (ii) Let $K_{1}, K_{2}, \cdots, K_{t}, L_{1}, L_{2}, \cdots, L_{t}$ are ideals of $R$ such that the sum $K_{1}+K_{2}$ $+\cdots+K_{t}$ is direct and $L_{i} \subseteq K_{i}$ for $1 \leq i \leq t$. Then $L_{1}+L_{2}+\cdots+L_{t} \leq_{e}$ $K_{1}+K_{2}+\cdots+K_{t} \Leftrightarrow L_{i} \leq_{e} K_{i}$ for $1 \leq i \leq t$.

Proof. (i) Assume that $L_{1} \leq_{e} K_{1}$ and $L_{2} \leq_{e} K_{2}$. Write $A_{1}=L_{1}+K_{2}$ and $A_{2}=$ $K_{1}+L_{2}$. We show that $A_{1} \leq_{e} K_{1}+K_{2}$. Let $0 \neq \mathrm{a} \in K_{1}+K_{2}$. Then a $=a_{1}+a_{2}$ for some $a_{1} \in K_{1}, a_{2} \in K_{2}$. If $a_{1}=0$, then a $\in A_{1}$ and hence $\left\langle a>\cap A_{1} \neq(0)\right.$. If $a_{1} \neq 0$, then since $L_{1} \leq_{e} K_{1}$ and $0 \neq<a_{1}>\subseteq K_{1}$ there exists $0 \neq x_{1} \in<a_{1}>$ $\cap L_{1}$. By Remark 2.6, there exists $x_{2} \in\left\langle a_{2}\right\rangle$ such that $x_{1}+x_{2} \in\left\langle a_{1}+a_{2}\right\rangle$. 
Since $x_{1} \neq 0,0 \neq x_{1}+x_{2} \in\left\langle a_{1}+a_{2}\right\rangle \cap A_{1}=\langle a\rangle \cap A_{1}$. Thus $A_{1} \leq_{e} K_{1}+K_{2}$. Similarly $A_{2} \leq_{e} K_{1}+K_{2}$. Since $L_{1}+L_{2}=A_{1} \cap A_{2}$ by Result 2.4(i), it follows that $L_{1}+L_{2}=A_{1} \cap A_{2} \leq_{e} K_{1}+K_{2}$.

Converse: Suppose that $L_{1}+L_{2} \leq_{e} K_{1}+K_{2}$. To show $L_{1} \leq_{e} K_{1}$, take $(0) \neq \mathrm{A}$ $\unlhd \mathrm{R}$ such that $\mathrm{A} \subseteq K_{1}$ and $L_{1} \cap \mathrm{A}=(0)$. Now $\mathrm{x} \in \mathrm{A} \cap\left(L_{1}+L_{2}\right) \Rightarrow \mathrm{x} \in \mathrm{A}$ and $\mathrm{x}$ $=l_{1}+l_{2}$ for some $l_{1} \in L_{1}$ and $l_{2} \in L_{2} \Rightarrow-l_{1}+\mathrm{x}=l_{2} \in\left(L_{1}+A\right) \cap L_{2} \subseteq K_{1} \cap$ $K_{2}=(0), l_{2}=0 \Rightarrow \mathrm{x}=l_{1} \in L_{1} \cap \mathrm{A}=(0)$. Therefore $\mathrm{A} \cap\left(L_{1}+L_{2}\right)=(0)$. Since $L_{1}+L_{2} \leq_{e} K_{1}+K_{2}$ we have that $\mathrm{A}=(0)$. Thus $L_{1} \leq_{e} K_{1}$. In a similar way, we can show $L_{2} \leq_{e} K_{2}$. The rest follows by using (i) and Mathematical induction on t.

Note 2.8. Consider ideals $\mathrm{A}, \mathrm{B}, \mathrm{C}$ of $\mathrm{R}$ as in Note 2.3 (ii) and (iii). Here $\mathrm{A} \oplus \mathrm{B}$ $\leq_{e} \mathrm{R}$ and $\mathrm{A} \oplus \mathrm{B} \subseteq \mathrm{C} \oplus \mathrm{B} \subseteq \mathrm{R}$. Using Result 2.4 (iv), we get that $\mathrm{A} \oplus \mathrm{B} \leq_{e} \mathrm{C} \oplus$ B. By Lemma 2.7, it follows that $\mathrm{A} \leq_{e} \mathrm{C}$. Note that $\mathrm{C}$ is a complement ideal which is also an essential extension of $\mathrm{A}$.

\section{Uniform ideals}

Definition 3.1. A non-zero ideal $\mathrm{I}$ of $\mathrm{R}$ is said to be uniform if $(0) \neq \mathrm{J} \unlhd \mathrm{R}$, and $\mathrm{J} \subseteq \mathrm{I} \Rightarrow \mathrm{J} \leq_{e} \mathrm{I}$.

Note 3.2. Let $R_{1}, R_{2}$ be two rings and $\mathrm{f}: R_{1} \rightarrow R_{2}$ is a ring isomorphism. I, $\mathrm{J} \unlhd$ $R_{1}$. Then $f^{-1}(I) \cap f^{-1}(J)=f^{-1}(I \cap J)$.

Theorem 3.3. (i) $I$ is an uniform ideal $\Leftrightarrow L \unlhd R, K \unlhd R, L \subseteq I, K \subseteq I, L \cap K=(0)$ $\Rightarrow L=(0)$ or $K=(0)$.

(ii) Let $R_{1}$ and $R_{2}$ be two rings and $f: R_{1} \rightarrow R_{2}$ be ring isomorphism. If $U$ is ideal of $R_{1}$, then $U$ is uniform in $R_{1} \Leftrightarrow f(U)$ is uniform in $R_{2}$.

(iii) Let $H$ and $K$ be two ideals of $R$ such that $H \cap K=(0)$. For an ideal $U$ of $R$ contained in $H$, we have that $U$ is uniform $\Leftrightarrow(U+K) / K$ is uniform in $R / K$.

(iv) If $U$ and $K$ are two ideals of $R$ such that $U \cap K=(0)$, then $U$ is uniform in $R \Leftrightarrow(U+K) / K$ is uniform in $R / K$.

Proof. (i) Let I be an uniform ideal of R. Suppose $\mathrm{L} \unlhd \mathrm{R}, \mathrm{K} \unlhd \mathrm{R}, \mathrm{L} \subseteq \mathrm{I}, \mathrm{K} \subseteq \mathrm{I}, \mathrm{L}$ $\cap \mathrm{K}=(0)$. Suppose $\mathrm{L} \neq(0)$. By our supposition, we have that $\mathrm{L} \leq{ }_{e} \mathrm{I}$. Since $\mathrm{L} \leq_{e} \mathrm{I}$ and $\mathrm{L} \cap \mathrm{K}=(0)$ by Note 2.3 (i), we have that $\mathrm{K} \cap \mathrm{I}=(0)$. Since $\mathrm{K} \subseteq \mathrm{I}$ it follows that $\mathrm{K}=\mathrm{K} \cap \mathrm{I}=(0)$. The other part of (i) is straight forward verification.

(ii) is direct verification.

(iii) Define $f: H \rightarrow(H+K) / K$ by $f(h)=h+K$. Then $f$ is a ring isomorphism. By (ii), we get that $U$ is uniform in $\mathrm{H} \Leftrightarrow f(U)=(U+K) / K$ is uniform in $(H+K) / K$. (iv) follows from (iii).

Remark 3.4. Let $K$ be an uniform ideal of $R$ and $L \unlhd R$ such that $L \subseteq K$. Then either $L=(0)$ or $L$ is uniform.

[Verification: Suppose $L \neq(0)$. Let $A \unlhd R, B \unlhd R$ such that $A \subseteq L, B \subseteq L$ and $A \cap B=(0)$. Since $A, B \subseteq L \subseteq K, A \cap B=(0)$ and $K$ is uniform, it follows 
that $A=(0)$ or $B=(0)$. This shows that $L$ is uniform.]

\section{Associative rings with finite dimension}

Definition 4.1. (i) We say that R has Finite Dimension on Ideals (FDI, in short) if $\mathrm{R}$ do not contain infinite number of non-zero ideals of $\mathrm{R}$ whose sum is direct.

(ii) Let $(0) \neq \mathrm{K} \unlhd \mathrm{R}$. We say that $\mathrm{K}$ has finite dimension on ideals of $\mathrm{R}$ (FDIR, in short) if $\mathrm{K}$ does not contain an infinite number of non-zero ideals of $\mathrm{R}$ whose sum is direct. It is clear that if $\mathrm{R}$ has FDI, then every ideal $\mathrm{K}$ of $\mathrm{R}$ has FDIR.

Theorem 4.2. $K$ has FDIR $\Leftrightarrow$ for any strictly increasing sequence $H_{1}, H_{2}, \cdots$ of ideals of $R$ contained in $K$, there is an integer $i$ such that $H_{k} \leq_{e} H_{k+1}$ for every $k$ $\geq i$.

Proof. Suppose K has FDIR. Take a strictly increasing sequence $H_{1} \subseteq H_{2} \cdots$ of ideals of R contained in K. In a contrary way, suppose that for every integer i there exists $\mathrm{k} \geq \mathrm{i}$ such that $H_{k}$ is not essential in $H_{k+1} \cdots \cdots$ (i)

Take $\mathrm{i}=1$. Then there exists $k_{1} \geq 1$ such that $H_{K_{1}}$ is not essential in $H_{K_{1}+1}$. Write $i_{2}=k_{1}+1$. Then by (i), there exists $k_{2} \geq i_{2}$ such that $H_{K_{2}}$ is not essential in $H_{K_{2}+1}$. Note that $k_{2} \geq k_{1}+1$. If we continue this process, then we get a subsequence $\left\{H_{K_{i}}\right\}_{i=1}^{\infty}$ of $\left\{H_{i}\right\}_{i=1}^{\infty}$ such that $H_{K_{i}}$ is not essential in $H_{K_{i}+1}$ and $k_{i+1} \geq k_{i}+1$. Since the sequence $H_{1} \subseteq H_{2} \cdots$ is increasing we have that $H_{K_{i+1}}$ $\supseteq H_{K_{i}+1}$. Since $H_{K_{i}}$ is not essential in $H_{K_{i}+1}$ and $H_{K_{i}+1} \subseteq H_{K_{i+1}}$ we have that $H_{K_{i}}$ is not essential in $H_{K_{i+1}}$. Thus we got a subsequence $\left\{H_{K_{i}}\right\}_{i=1}^{\infty}$ of $\left\{H_{i}\right\}_{i=1}^{\infty}$ such that $H_{K_{i}}$ is not essential in $H_{K_{i+1}}$ for all i. Write $B_{i}=H_{K_{i}}$ for $\mathrm{i} \geq 1$. Now $\left\{B_{i}\right\}_{i=1}^{\infty}$ is an increasing sequence of ideals of $\mathrm{R}$ contained in $\mathrm{K}$ such that $B_{i}$ is not essential in $B_{i+1}$. Now for each i there exists a non-zero ideal $A_{i}$ of $\mathrm{R}$ contained in $\mathrm{K}$ such that $A_{i} \subseteq B_{i+1}$ and $B_{i} \cap A_{i}=(0)$. Now we verify that the sum $\sum_{i=1}^{\infty} A_{i}$ is a direct sum of infinite number of non-zero ideals $\left\{A_{i}\right\}_{i=1}^{\infty}$. Let $x_{1} \in A_{i_{1}}, x_{2} \in$ $A_{i_{2}}, \cdots, x_{n} \in A_{i_{n}}$ such that $x_{1}+x_{2}+\cdots+x_{n}=0$. With out loss of generality, we may assume that $i_{1}<i_{2}<\cdots<i_{n}$. Also we can suppose that $x_{n} \neq 0$.

Now $A_{i_{1}} \subseteq B_{i_{1}+1} \subseteq \cdots \subseteq B_{i_{n}}, A_{i_{2}} \subseteq B_{i_{2}+1} \subseteq \cdots \subseteq B_{i_{n}}, \cdots, A_{i_{n-1}} \subseteq B_{i_{n-1}+1} \subseteq$ $\cdots \subseteq B_{i_{n}}$

$x_{1}+x_{2}+\cdots+x_{n-1} \in A_{i_{1}}+A_{i_{2}}+\cdots+A_{i_{n-1}} \subseteq B_{i_{n}}$.

Now $\left(A_{i_{1}}+A_{i_{2}}+\cdots+A_{i_{n-1}}\right) \cap A_{i_{n}} \subseteq B_{i_{n}} \cap A_{i_{n}}=(0) \Rightarrow x_{1}+x_{2}+\cdots+x_{n-1}=$ $-x_{n} \in\left(A_{i_{1}}+A_{i_{2}}+\cdots+A_{i_{n-1}}\right) \cap A_{i_{n}}=(0) \Rightarrow x_{n}=0$, a contradiction. Hence the sum $\sum_{i=1}^{\infty} A_{i}$ is a direct sum of infinite number of non-zero ideals of $\mathrm{R}$ contained in $\mathrm{K}$, a contradiction to (i). This completes the proof for (i) $\Rightarrow$ (ii).

(ii) $\Rightarrow$ (i): Suppose (ii). We have to verify that K does not contain a direct sum of infinite number of non-zero of ideals of $\mathrm{R}$. In a contrary way, suppose that $\mathrm{K}$ contains a direct sum of infinite number of non-zero of ideals $\left\{I_{i}\right\}_{i=1}^{\infty}$. Write $J_{n}=$ $I_{1}+I_{2}+\cdots+I_{n}$ for $\mathrm{n} \geq 1$. Then $J_{1} \subseteq J_{2} \subseteq \cdots$. Since $(0) \neq I_{n} \subseteq J_{n+1}$ and $J_{n}$ $\cap I_{n+1}=(0)$, it follows that $J_{n}$ is not essential in $J_{n+1}$. This is true for all $\mathrm{n} \geq$ 1. Thus we arrived at a strictly increasing sequence $J_{1} \subseteq J_{2} \subseteq \cdots$ of ideals of $\mathrm{R}$ contained in $\mathrm{K}$ such that $J_{i}$ is not essential in $J_{i+1}$ for $\mathrm{i} \geq 1$, a contradiction to the 
assumed condition (ii). This completes the proof.

Lemma 4.3. If $R$ has FDI and $(0) \neq K \unlhd R$, then $K$ contains an uniform ideal of $R$.

Proof. In a contrary way, suppose $\mathrm{K}$ contains no uniform ideal of $\mathrm{R}$. Then $\mathrm{K}$ is not uniform ideal of $\mathrm{R}$. So there exist $(0) \neq K_{1} \unlhd \mathrm{R}$ and $(0) \neq L_{1} \unlhd \mathrm{R}$ such that $K_{1} \cap L_{1}=(0)$, and $K_{1}+L_{1} \subseteq \mathrm{K}$ (by Theorem 3.3(i)). Now $L_{1}$ is not uniform and so there exist $(0) \neq K_{2} \unlhd \mathrm{R},(0) \neq L_{2} \unlhd \mathrm{R}$ such that $K_{2} \cap L_{2}=(0)$ and $K_{2}+L_{2} \subseteq L_{1}$. If we continue this process, we get two infinite sequences $\left\{K_{i}\right\}_{i=1}^{\infty},\left\{L_{i}\right\}_{i=1}^{\infty}$ of non-zero ideals of $\mathrm{R}$ such that $K_{i} \cap L_{i}=(0)$ for each i and $K_{i}+L_{i} \subseteq L_{i-1}$ for $\mathrm{i} \geq 2$. Also note that $L_{1} \supseteq L_{2} \supseteq \cdots$. The sum $\sum_{i=1}^{\infty} K_{i}$ is an infinite direct sum of non-zero ideals of R. [Verification: In contrary way, suppose that there exist non-zero elements $x_{1} \in K_{i_{1}}, x_{2} \in K_{i_{2}}, \cdots, x_{n} \in K_{i_{n}}$ such that $x_{1}+x_{2}+\cdots+x_{n}=0$ where $i_{1}<i_{2}<\cdots<i_{n} . x_{n} \in K_{i_{n}} \subseteq L_{\left(i_{n}\right)-1} \subseteq \cdots \subseteq L_{i_{1}}, x_{n-1}$ $\in K_{i_{n-1}} \subseteq L_{\left(i_{n-1}\right)-1} \subseteq \cdots \subseteq L_{i_{1}}, x_{2} \in K_{i_{2}} \subseteq L_{\left(i_{2}\right)-1} \subseteq L_{i_{1}}$. Now $x_{2}+x_{3}+\cdots+x_{n}$ $\in L_{i_{1}}$. Since $x_{1} \in K_{i_{1}}$ and $x_{2}+x_{3}+\cdots+x_{n} \in L_{i_{1}}$, it follows that $-x_{1}=x_{2}+\cdots+x_{n}$ $\in K_{i_{1}} \cap L_{i_{1}}=(0)$ and so $x_{1}=0$, a contradiction]. This is a contradiction to the fact that $\mathrm{R}$ has FDI. This completes the proof.

Theorem 4.4. Suppose $0 \neq H \unlhd R$ and $H$ has FDIR. Then the following conditions hold.

(i) (Existence) There exist uniform ideals $U_{1}, U_{2}, \cdots, U_{n}$ of $R$ whose sum is direct and essential in $H$;

(ii) If $V_{i}, 1 \leq i \leq k$ are uniform ideals of $R$, whose sum is direct and essential in $H$, then $k \leq n$.

(iii) (Uniqueness) If $V_{i}, 1 \leq i \leq k$ are uniform ideals of $R$ whose sum is direct and essential in $H$, then $k=n$.

Proof. (i) Suppose $\mathrm{H}$ has FDIR. In a contrary way, suppose that for any finite number of uniform ideals $U_{i}, 1 \leq \mathrm{i} \leq \mathrm{n}$ whose sum is direct, the sum $\sum U_{i}$ is not essential in H. By Lemma 4.3, $\mathrm{H}$ contains an uniform ideal $U_{1}$. Then $U_{1}$ is not essential in $\mathrm{H}$. So there exists $0 \neq H_{1} \unlhd \mathrm{R}$ such that $H_{1} \subseteq \mathrm{H}$ with $U_{1} \cap H_{1}=(0)$. Again by using Lemma 4.3, we conclude that $H_{1}$ contains a uniform ideal $U_{2}$. Now the sum $U_{1}+U_{2}$ is a direct sum of two uniform ideals. So $U_{1}+U_{2}$ is not essential in $\mathrm{H}$. This means, there exists $(0) \neq H_{2} \unlhd \mathrm{R}, H_{2} \subseteq \mathrm{H}$ such that $\left(U_{1}+U_{2}\right) \cap H_{2}$ $=(0)$. Again by using Lemma 4.3, we get an uniform ideal $U_{3} \subseteq H_{2}$. Now the sum $U_{1}+U_{2}+U_{3}$ is direct. If we continue this process, we get an infinite strictly increasing chain $U_{1} \subset\left(U_{1}+U_{2}\right) \subset\left(U_{1}+U_{2}+U_{3}\right) \cdots$ of ideals of $\mathrm{R}$ such that $U_{1} \oplus U_{2} \oplus \cdots \oplus U_{s}$ is not essential in $U_{1} \oplus U_{2} \oplus \cdots \oplus U_{s} \oplus U_{s+1}$ for all $\mathrm{s} \geq 1$. By Theorem 4.2, it follows that $\mathrm{H}$ has no FDIR, a contradiction to our assumption. Hence there exist uniform ideals $U_{i}, 1 \leq \mathrm{i} \leq \mathrm{n}$ in $\mathrm{R}$ whose sum $U_{1}+U_{2}+\cdots+U_{n}$ is direct and essential in $\mathrm{H}$.

(ii) Suppose $V_{i}, 1 \leq \mathrm{i} \leq \mathrm{k}$ are uniform ideals of $\mathrm{R}$ whose sum is direct and $V_{1}+V_{2}+$ $\cdots+V_{k} \subseteq \mathrm{H}$. Write $K_{1}=V_{2} \oplus V_{3} \oplus \cdots \oplus V_{k}$. Since $K_{1}$ is not essential in H, there exists $\mathrm{i}(1 \leq \mathrm{i} \leq \mathrm{n})$ such that $K_{1} \cap U_{i}=(0)$. Without loss of generality, we may 
assume that $K_{1} \cap U_{1}=(0)$. Note that the sum $U_{1}+V_{2}+\cdots+V_{k}$ is direct. Write $K_{2}=U_{1} \oplus V_{3} \oplus \cdots \oplus V_{k}$. Since $K_{2}$ is not essential in $\mathrm{H}$ there exists an $\mathrm{i}(2 \leq \mathrm{i} \leq$ n) such that $K_{2} \cap U_{i}=(0)$. We may suppose that $K_{2} \cap U_{2}=(0)$. Now the sum $U_{1}+U_{2}+V_{3}+\cdots+V_{k}$ is direct. If we continue this process, we can replace each $V_{j}$ $(1 \leq \mathrm{j} \leq \mathrm{k})$ by some $U_{i}(1 \leq \mathrm{i} \leq \mathrm{n})$. From this discussion, we can conclude $\mathrm{k} \leq \mathrm{n}$. (iii) In (ii) we verified that $\mathrm{k} \leq \mathrm{n}$. Similarly, we can verify that $\mathrm{n} \leq \mathrm{k}$. Hence $\mathrm{k}=$ n.

As a consequence of this result we have the following Corollary.

Corollary 4.5. If $R$ is a ring with FDI, then the following (i) - (ii) are true: (i) (Existence) There exist uniform ideals $U_{1}, U_{2}, \cdots U_{n}$ in $R$ whose sum is direct and essential in $R$;

(ii) (Uniqueness) If $V_{i}, 1 \leq i \leq k$, are uniform ideals of $R$ whose sum is direct and essential in $R$, then $k=n$.

Definition 4.6. The number $n$ of the above Theorem is independent of the choice of the uniform ideals. This number $\mathrm{n}$ is called the dimension of $\mathrm{H}$, and is denoted by $\operatorname{dim} \mathrm{H}$.

Acknowledgment. The first and second authors are thankful to the UGC (New Delhi) for its financial assistance under the grant No. F. 8-8/2004 (SR), dated 2912-2003.

\section{References}

[1] M. R. Adhikari, Groups, Rings and Modules with Applications, University Press (India) Ltd., Hyderabad, India, 1999.

[2] A. W. Goldie, The Structure of Noetherian Rings, Lectures on Rings and Modules, Springer-Verlag, New York, 1972.

[3] I. N. Herstein, Topics in Algebra (Second Edition), Wiley Eastern Limited, New Delhi, 1998.

[4] Y. V. Reddy and Bh. Satyanarayana, A Note on Modules, Proc. of the Japan Academy, 63-A(1987), 208-211.

[5] Bh. Satyanarayana, A note on E-direct and S-inverse Systems, Proc. of the Japan Academy, 64-A(1988), 292-295.

[6] Bh. Satyanarayana, K. Syam Prasad and D. Nagaraju, A Theorem on Modules with Finite Goldie Dimension, Soochow J. Maths 32(2)(2006), 311-315. 